\title{
Incidence of acute kidney injury in bone marrow transplant patients; investigation of consequences within one to three years
}

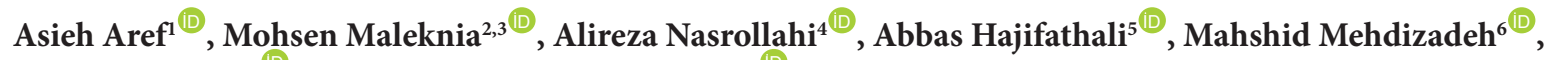 \\ Hashem Kazemi $^{2}$, Mohammad Hassan Ghadiani ${ }^{*}{ }^{*}$ \\ ${ }^{1}$ Department of Internal Medicine, Chronic Renal Failure Research Center, Ahvaz Jundishapur University of Medical Sciences, Ahvaz, Iran \\ ${ }^{2}$ Thalassemia and Hemoglobinopathy Research Center, Health Research Institute, Ahvaz Jundishapur University of Medical Sciences, Ahvaz, Iran \\ ${ }^{3}$ Student Research Committee, Ahvaz Jundishapur University of Medical Sciences, Ahvaz, Iran \\ ${ }^{4}$ Department of Nephrology, Taleghani Hospital, Shahid Beheshti University of Medical Sciences, Tehran, Iran \\ ${ }^{5}$ Department of Medical Genetics, Shahid Beheshti University of Medical Sciences, Tehran, Iran \\ ${ }^{6}$ Hematopoietic Stem Cell Research Center, Shahid Beheshti University of Medical Sciences, Tehran, Iran \\ ${ }^{7}$ Urology and Nephrology Research Center, Shahid Beheshti University of Medical Sciences, Tehran, Iran
}

\section{ART I C L E IN F O}

\section{Article Type:}

Original

\section{Article History:}

Received: 10 August 2020

Accepted: 26 September 2020

Published online: 29 October 2020

\section{Keywords:}

Acute kidney injury

Bone marrow transplantation

Allogeneic

Autologous

\begin{abstract}
A B S T R A C T
Introduction: Previous studies have demonstrated that acute kidney injury (AKI) is a serious complication following hematopoietic stem cell transplantation (HSCT). The incidence of AKI in association with HSCT varies considerably because of several definitions for AKI.

Objectives: In this study, we determined the rate of AKI after bone marrow transplantation (BMT) and its effects on patients' outcomes according to modern definitions of AKI to conclude whether all these criteria can be useful for predicting AKI occurrence after BMT or not.

Patients and Methods: We conducted a retrospective study of 271 patients undergoing HSCT, and after obtaining written informed consent from all patients, the required information was reviewed. AKI was defined according to RIFLE, KDIGO, and AKIN criteria. Renal function was assessed by calculating creatinine clearance, urine output, and estimated glomerular filtration rate (eGFR), determined through the MDRD equation.

Results: Allogeneic and autologous transplantations were performed on $38(14.02 \%)$ and 233 (85.97\%) patients, respectively. According to the RIFLE criteria, 96 patients (35.42\%) suffered from AKI, and based on AKIN, and KDIGO criteria, 101 patients (37.26\%) were afflicted with it after BMT. The one-year mortality rate in allogeneic transplant patients with a history of AKI was $30.43 \%$ and $53.33 \%$ in patients without a history of AKI. The three-year mortality rate in allogeneic transplant patients with and without a history of AKI was $52.17 \%$ and $73.33 \%$ respectively, which showed no statistically significant difference. The three-year mortality rate in autologous transplant patients with and without a history of AKI was $60.27 \%$ and $22.5 \%$, respectively. Conclusion: The one-year and three-year mortality rates, survival of patients, and AKI's diagnosis were similar in all three criteria. Therefore, all these criteria can be useful for the prediction of AKI occurrence after BMT.
\end{abstract}

Implication for health policy/practice/research/medical education:

Previous studies have demonstrated that acute kidney injury (AKI) is a serious complication following hematopoietic stem cell transplantation (HSCT). The incidence of AKI in association with HSCT varies considerably because of several definitions for AKI. In this study, we determine the rate of kidney injury after bone marrow transplantation (BMT) and its effects on patients' outcomes according to modern definitions of AKI. This study enrolled 271 patients (including 158 males and 113 females) aged above 18 years (with a mean age of $38.02 \pm 8$ years) undergoing HSCT. AKI was defined according to RIFLE, KDIGO, and AKIN criteria. In the present study, the one-year and three-year mortality rates, survival of patients, and AKI's diagnosis were similar in all three KDIGO, RIFLE, and AKIN criteria.

Please cite this paper as: Aref A, Maleknia M, Nasrollahi A, Hajifathali A, Mehdizadeh M, Kazemi H, Ghadiani MH. Incidence of acute kidney injury in bone marrow transplant patients; investigation of consequences within one to three years. J Renal Inj Prev. 2021; 10(2): e10. doi: 10.34172/jrip.2021.10. 


\section{Introduction}

Bone marrow transplantation (BMT), also known as hematopoietic stem cell transplantation (HSCT), is an important curative approach for malignant and hematologic disorders (1). However, morbidity and mortality rates are continually increasing in these patients, especially within the first three months posttransplantation. HSCs used in this procedure are obtained from the peripheral blood as a stem cell source or from bone marrow and subsequently injected into the patient after a course of chemotherapy preparation (2). Like any other treatment, BMT has acute and chronic side effects depending on the type of transplant, therapeutic agents, irradiation, and graft versus host disease (GVHD) (3). Acute kidney injury (AKI) and long-term renal dysfunction are common and severe complications of BMT, highly variable after BMT, and mainly influenced by the definition of AKI and the study population (4). It is noteworthy that almost $25-50 \%$ of patients subject to BMT develop AKI. Furthermore, more than $20 \%$ of all survivors in the long-term follow-up develop chronic kidney disease (5-7). There are several causes for AKI that include (1) chemotherapy, high-dose radiation with fluid loss through diarrhea or vomiting, nephrotoxic drugs such as antimicrobials, calcineurin inhibitors or sepsis (2), BMT-specific factors like hepatic veno-occlusive disease, thrombotic microangiopathy (TMA), marrow infusion toxicity and GVHD (8). The frequency of AKI and kidney dysfunction is directly related to the BMT method. The type of BMT (allogeneic/autologous) is more important than co-factors, such as co-morbidities, advanced age, or high serum creatinine $(\mathrm{sCr})$ at baseline (9). In allogeneic BMT, approximately $92 \%$ of patients develop kidney dysfunction with an overall mortality of $58 \%$ compared to a $56 \%$ incidence of renal dysfunction among autologous BMT patients with an overall mortality of $7 \%(10,11)$. In many patients, kidney injury is transient and renal function is reversible. However, the same acute kidney dysfunction can affect the prognosis of patients independently. In contrast, the exact determination of kidney function in BMT will affect the dose setting of prescription drugs. Today a standardized classification representing the AKI status has been widely applied in clinical trials (12). Initially, the RIFLE (Risk, Injury, Failure, Loss, End-Stage Kidney Disease) criteria were proposed by the Acute Dialysis Quality Initiative group (ADQI). After that, the Acute Kidney Injury Network, known as the AKIN Network classification system, was developed $(13,14)$.

\section{Objectives}

Recently, the KDIGO (Kidney Disease: Improving Global Outcomes) proposed a new definition of AKI based on both the AKIN and RIFLE criteria. Besides, Studies showed that KIDGO is more capable of predicting hospitalized patients' prognosis than the other two criteria $(15,16)$. Accordingly, evaluating the severity and progression of AKI following BMT using AKI-related descriptions such as definition and staging of AKI based on RIFLE, renal disease, namely Improving Global Outcomes (KDIGO), and AKIN can help control and improve patients' conditions (8). In this study, due to the mortality/survival rates of transplanted patients, we used the RIFLE, AKIN and, KDIGO Criteria for the diagnosis and prognosis of AKI and compared the performance of these criteria in the prediction of post-transplant outcomes.

\section{Patients and Methods \\ Study design}

This retrospective study was conducted between March 2007 and March 2013 on 271 patients at the bone marrow transplant center, Taleghani hospital of Tehran, Iran. Two hundred and seventy-one patients, including 158 males (58.3\%) and 113 females (41.7\%) with a mean age of 38.02 \pm 8 and an age range of 19-67 years were studied. After obtaining written informed consent from all patients, the required information, including age, gender, routine blood, and non-blood tests were reviewed. All patients undergoing allogeneic or autologous transplantation over 18 years of age were recruited in this study, regardless of their underlying disease. The inclusion criteria in this study were patients over 18 years, hospitalization at the BMT center, receiving BMT at the time of admission, the possibility of daily blood-test results, and a urine volume chart. Exclusion criteria were incomplete information in the transplant patient records, and transplant failure at the time of admission. All patients were subjected to complete evaluation before transplantation, including history and physical examination, routine blood and non-blood tests, and renal function assessment by calculating creatinine clearance as well as estimated glomerular filtration rate (eGFR) by MDRD equation, as modified by the Japanese society of nephrology; eGFR $\left(\mathrm{ml} / \mathrm{min} / 1.73 \mathrm{~m}^{2}\right)=194 \times \mathrm{Cr}$ $-1.094 \times$ age $^{-0.287} \times 0.739$ (if female) (22). Besides, previous diseases and medical records of the patients were recorded. The type of preparation regimen, length of hospital stays, and complications during hospitalization were also carefully studied.

\section{Preparation procedure}

The conditioning regimen was prescribed in patients subject to autologous transplantation following five days using granulocyte-colony stimulating factor treatment for mobilization and harvest of circulating hematopoietic stem cells. After being assured of appropriate donors, the patients underwent preparation in terms of their underlying disease. In allogeneic transplantation, bone marrow and peripheral blood stem cells from related donors were used as the source of hematopoietic progenitors. None of the patients from this center was prepared with radiotherapy 
regimens or total body irradiation.

\section{Patient monitoring}

Patients were fully hospitalized after transplantation until recovery from major complications. They were monitored during their entire hospital stay for several complications, including frequent monitoring of biochemical tests, control of infection (documented by positive blood cultures), urinary output, blood pressure (defined as systolic blood pressure $<90 \mathrm{~mm} \mathrm{Hg}$ ), and vital organ function (liver and gastrointestinal GVHD). Frequent measurements of weight and vital signs were also performed during this period. Kidney function was assessed daily in terms of urinary output and sCr level.

\section{Determination of AKI}

AKI within the first 100-days after BMT was defined according to the sCr and/or eGFR criteria. All three definitions of KDIGO, RIFLE, and AKIN were used for the determination of AKI. (16). Patients were assessed during daily hospitalization. If AKI occurred, they were graded according to any of the descriptions during the first three months after transplantation and followed up three years after it. The patients' clinical outcome was studied in the first three months, first year, and the end of the third year. According to the KDIGO criteria, we determined the stage or severity of AKI by urine output measurement, eGFR, and sCr levels as follows;

Stage 1) sCr level increase of $\geq 0.3 \mathrm{mg} / \mathrm{dL}$ within 48 hours or increase in $\mathrm{sCr}$ to $\geq 1.5$ times of baseline, which occurred within seven days and urine output decrease of $<0.5 \mathrm{~mL} / \mathrm{kg} / \mathrm{h}$ for 6-hours. Stage 2) Reduction in urine output $<0.5 \mathrm{~mL} / \mathrm{kg} / \mathrm{h}$ for 12 -hour or increase in $\mathrm{sCr}$ to 2.0-2.9 times of baseline. Stage 3) Reduction in urine output $<0.3 \mathrm{~mL} / \mathrm{kg} / \mathrm{h}$ for 24 hours, an increase in $\mathrm{sCr}$ to $\geq 4.0 \mathrm{mg} / \mathrm{dl}$ or decrease in eGFR to $<35 \mathrm{ml} / \mathrm{min}$ per 1.73 $\mathrm{m}^{2}$. We determined the stage or severity of AKI based on the AKIN criteria by urine output measurement and sCr levels; Stage 1) Reduction in urine output $<0.5 \mathrm{~mL} /$ $\mathrm{kg} / \mathrm{h}$ for 6 hours or sCr increase of $\geq 0.3 \mathrm{mg} / \mathrm{dL}$. Stage 2) Reduction in urine output $<0.5 \mathrm{~mL} / \mathrm{kg} / \mathrm{h}$ for 12 hours or increase in $\mathrm{sCr}$ to $>2-3$ times baseline. Stage 3) Reduction in urine output $<0.3 \mathrm{~mL} / \mathrm{kg} / \mathrm{h}$ for 24 hours or increase in $\mathrm{sCr}$ to $\geq 4.0 \mathrm{mg} / \mathrm{dL}$. Besides, we used RIFLE criteria for the definition of the AKI stage through changes in sCr levels, GFR, or a decrease in urine output. Risk (R): Reduction in urine output $<0.5 \mathrm{~mL} / \mathrm{kg} / \mathrm{h}$ for 6-hour, increased in $\mathrm{sCr}$ to 1.5 times baseline or decrease in GFR $\geq 25 \%$. Injury (I): Doubling of sCr, reduction in urine output $<0.5 \mathrm{~mL} / \mathrm{kg} / \mathrm{h}$ for 12 hours or decrease in GFR $\geq 50 \%$. Failure (F): Urine output below $0.3 \mathrm{~mL} / \mathrm{kg} / \mathrm{h}$ for 24 hours, tripling of $\mathrm{sCr}$ (or creatinine $>355 \mu \mathrm{mol} / \mathrm{L}$ ) or decrease in GFR $\geq 75 \%$. Loss (L): Complete loss of kidney function for more than four weeks (or persistent AKI). End-stage renal disease (E): the need for kidney replacement therapy for $>3$ months. In this study, stages "L" (complete loss of kidney function $>4$ weeks) and "E" (the need for kidney replacement therapy for $>3$ months) was not included in the analysis.

\section{Ethical issues}

The research followed the tenets of the Declaration of Helsinki. The institutional ethical committee at Shahid Beheshti University of Medical Sciences approved all study protocols (\#IR.SBUMS.UNRC.REC.94.201). Accordingly, written informed consent was taken from all participants before any intervention. This study was extracted from nephrology fellowship thesis of Asieh Aref at this university (Thesis \#258).

\section{Statistical analysis}

This study's variables included quantitative and qualitative data and the former were reported as mean \pm standard deviation (mean $\pm \mathrm{SD}$ ). All the statistical analyses were processed by SPSS software (statistical package for the social sciences, version 22) and $P$ value $<0.05$ was considered statistically significant. For the determination of each AKI category association with mortality, we used Cox regression analysis in this study, followed by adjusted hazard ratio (HR) calculation and 95\% confidence intervals. A chi-square test was used to analyze qualitative data. A student t-test was also used to analyze the parametric variables for comparing continuous variables between different groups of AKI patients. Additionally, the Kaplan-Meier method was used to assess one-year and three-year survival of transplant patients.

\section{Results}

In this research, 271 patients, including 158 males (58.3\%) and 113 females (41.7\%) were studied. Allogeneic and autologous transplantations were performed on 38 (14.02\%) and 233 patients (85.97\%), respectively. According to RIFLE criteria, 96 patients (35.42\%) were afflicted with AKI, and based on AKIN and KDIGO criteria, 101 patients (37.26\%) suffered from AKI. Concerning the type of transplantation, 23 allogeneic patients $(60.52 \%)$ suffered from AKI according to RIFLE criteria, while 24 patients (63.15\%) were afflicted with AKI based on AKIN and KDIGO criteria. As indicated by the RIFLE criterion, 73 autologous patients (31.33\%) suffered from AKI, and based on AKIN and KDIGO criteria, 77 autologous patients (33.04\%) were distressed with AKI (Table 1). The severity of kidney injury based on the three criteria among allogeneic and autologous transplants is presented in Table 2. According to RIFLE criteria, 14 patients $(60.86 \%)$ were in stage $\mathrm{R}$, six patients $(26.08 \%)$ were in stage 1 , and three patients (13.04\%) in stage $\mathrm{F}$, while in autologous transplantation, 50 patients (68.49\%) were in stage R, 20 patients (27.39\%) were in stage 1 and $3(4.1 \%)$ in stage F. Based on AKIN and KDIGO criteria in allogeneic transplantation, 15 patients $(62.5 \%)$ were 
Table 1. The incidence of AKI during the first three months after allogeneic/autologous bone marrow transplantation based on any of the RIFLE, AKIN, and KDIGO criteria

\begin{tabular}{|c|c|c|c|c|c|c|c|}
\hline Criteria & Patients & AKI & Type of transplantation & Number (\%) & AKI occur & & $P$ value \\
\hline \multirow{4}{*}{ RIFLE } & \multirow{4}{*}{271} & \multirow{4}{*}{$96(35 / 42 \%)$} & Allogenic & $38(14.02)$ & AKI & $23(60.52 \%)$ & 0.10 \\
\hline & & & & & Non- AKI & $15(39.47 \%)$ & 0.11 \\
\hline & & & Autologous & 233 (85.97) & AKI & $73(31.33 \%)$ & 0.10 \\
\hline & & & & & Non- AKI & 160 (68.66\%) & 0.13 \\
\hline \multirow{4}{*}{ AKIN } & \multirow{4}{*}{271} & \multirow{4}{*}{$101(37 / 26 \%)$} & Allogenic & $38(14.02)$ & AKI & $24(63.15 \%)$ & 0.11 \\
\hline & & & & & Non- AKI & $14(36.84 \%)$ & 0.10 \\
\hline & & & Autologous & $233(85.97)$ & AKI & 77 (33.04\%) & 0.56 \\
\hline & & & & & Non- AKI & $156(66.95 \%)$ & 0.67 \\
\hline \multirow{4}{*}{ KDIGO } & \multirow{4}{*}{271} & \multirow{4}{*}{$101(37 / 26 \%)$} & Allogenic & $38(14.02)$ & AKI & $24(63.15 \%)$ & 0.10 \\
\hline & & & & & Non- AKI & $14(36.84 \%)$ & 0.81 \\
\hline & & & Autologous & $233(85.97)$ & AKI & 77 (33.04\%) & 0.25 \\
\hline & & & & & Non- AKI & 156 (66.95\%) & 0.67 \\
\hline
\end{tabular}

in stage 1, 6 patients (25\%) in stage 2, and $3(12.5 \%)$ in stage 3, while in autologous transplantation, 55 patients $(71.42 \%)$ were in stage 1,19 patients $(24.67 \%)$ were in stage 2 , and 3 patients (3.89\%) in stage 3 . Table 3 shows the mortality rates of patients with and without AKI in the first year after transplantation by separating AKI criterion, severity, and association of mortality with relapse or non-relapse. Similarly, Table 4 illustrates the mortality of patients with and without AKI within the third year after transplantation based on the RIFLE, AKIN, and KDIGO

Table 2. The severity of AKI based on RIFLE, AKIN and KDIGO criteria in allogeneic and autologous transplant patients

\begin{tabular}{|c|c|c|c|c|}
\hline Criteria & $\begin{array}{l}\text { Type of } \\
\text { transplantation }\end{array}$ & AKI stage & $\begin{array}{c}\text { Number of } \\
\text { patients }\end{array}$ & $\%$ \\
\hline \multirow{6}{*}{ RIFLE } & \multirow[t]{3}{*}{ Allogenic } & $\mathrm{R}$ & 14 & 60.86 \\
\hline & & I & 6 & 26.08 \\
\hline & & $F$ & 3 & 13.04 \\
\hline & \multirow[t]{3}{*}{ Autologous } & $\mathrm{R}$ & 50 & 68.49 \\
\hline & & 1 & 20 & 27.39 \\
\hline & & $F$ & 3 & 4.1 \\
\hline \multirow{6}{*}{ AKIN } & \multirow[t]{3}{*}{ Allogenic } & 1 & 15 & 62.5 \\
\hline & & 2 & 6 & 25 \\
\hline & & 3 & 3 & 12.5 \\
\hline & \multirow[t]{3}{*}{ Autologous } & 1 & 55 & 71.42 \\
\hline & & 2 & 19 & 24.67 \\
\hline & & 3 & 3 & 3.89 \\
\hline \multirow{6}{*}{ KDIGO } & \multirow[t]{3}{*}{ Allogenic } & 1 & 15 & 62.5 \\
\hline & & 2 & 6 & 25 \\
\hline & & 3 & 3 & 12.5 \\
\hline & \multirow[t]{3}{*}{ Autologous } & 1 & 55 & 71.42 \\
\hline & & 2 & 19 & 24.67 \\
\hline & & 3 & 3 & 3.89 \\
\hline
\end{tabular}

criteria. The one-year survival rate of transplant patients with AKI is shown in Table 5 (concerning RIFLE, AKIN, and KDIGO criteria). Besides, Table 6 depicts the threeyear survival rates of transplant patients based on the same three criteria. The frequency of underlying malignancies (such as multiple myeloma, Hodgkin's lymphoma, and non-Hodgkin's lymphoma (NHL), acute myelogenous leukemia, and acute lymphoblastic leukemia that may have contributed to relapse mortality after autologous/ allogeneic BMT is summarized in Table 7. In this regard, the prevalence of deaths and survival of transplanted patients were analyzed according to the relapse mortality caused by underlying malignancies, AKI, as well as nonrelapse mortality. Therefore, the results of this study may be slightly different compared to other studies. Overall, 15 allogeneic patients (39.47\%) died at the end of the first year, and 23 allogeneic patients $(60.52 \%)$ were deceased at the end of the third year. This means that the survival rate of allogeneic transplant patients at the end of the first and third years in this study was $65.5 \%$ and $39.4 \%$, respectively. Generally, at the end of the first year, 15 autologous patients $(6.43 \%)$ perished, and 80 patients $(34.33 \%)$ died at the end of the third year. As a result, the survival rate of autologous transplant patients at the end of the first and third years in this research was $93.56 \%$ and $65.66 \%$, respectively. The one-year mortality of allogeneic transplant patients with a history of AKI was 30.43\%, which was $53.33 \%$ in patients without a history of AKI. The three-year mortality rate among allogeneic transplant patients with a history of AKI was $52.17 \%$, and it was $73.33 \%$ in patients without a history of AKI, indicating no statistically significant difference (Table 4 ). The one-year mortality in autologous transplant patients with a history of AKI was $13.69 \%$, which was $3.12 \%$ in those without a history of AKI. Three-year mortality in autologous transplant patients with and without a history of AKI 
Table 3. Comparison of the mortality related/unrelated to the one-year recurrence between transplant patients with or without AKI based on all RIFLE, AKIN, and KDIGO criteria

\begin{tabular}{|c|c|c|c|c|c|c|}
\hline Criteria & AKI/No AKI & RM/NRM & AKI stage & Allogeneic patients' mortality (\%) & Autologous patients' mortality (\%) & $P$ value \\
\hline \multirow{8}{*}{ RIFLE } & \multirow{6}{*}{ AKI } & \multirow{3}{*}{ RM } & Risk & 7.14 & 2 & 0.1 \\
\hline & & & Injury & 16.66 & 12 & 0.2 \\
\hline & & & Failure & 33.33 & 33.33 & 0.1 \\
\hline & & \multirow{3}{*}{ NRM } & Risk & 7.14 & 6 & 0.2 \\
\hline & & & Injury & 16.66 & 5 & 0.1 \\
\hline & & & Failure & 66.66 & 33.33 & 0.1 \\
\hline & \multirow{2}{*}{ Non- AKI } & $\mathrm{RM}$ & & 26.66 & 1.25 & 0.15 \\
\hline & & NRM & & 26.66 & 1.87 & 0.2 \\
\hline \multirow{8}{*}{ AKIN } & \multirow{6}{*}{ AKI } & \multirow{3}{*}{ RM } & Stage 1 & 6.66 & 1.81 & 0.1 \\
\hline & & & Stage 2 & 16.66 & 15.78 & 0.13 \\
\hline & & & Stage 3 & 33.33 & 33.33 & 0.13 \\
\hline & & \multirow{3}{*}{ NRM } & Stage 1 & 6.66 & 5.45 & 0.12 \\
\hline & & & Stage 2 & 16.66 & 5.26 & 0.13 \\
\hline & & & Stage 3 & 66.66 & 33.33 & 0.13 \\
\hline & \multirow{2}{*}{ Non- AKI } & RM & & 28.57 & 1.28 & 0.1 \\
\hline & & NRM & & 28.57 & 1.92 & 0.12 \\
\hline \multirow{8}{*}{ KDIGO } & \multirow{6}{*}{ AKI } & \multirow{3}{*}{ RM } & Stage 1 & 6.25 & 1.78 & 0.13 \\
\hline & & & Stage 2 & 20 & 16.66 & 0.12 \\
\hline & & & Stage 3 & 33.33 & 33.33 & 0.13 \\
\hline & & \multirow{3}{*}{ NRM } & Stage 1 & 12.50 & 10.71 & 0.1 \\
\hline & & & Stage 2 & 40 & 5.55 & 0.12 \\
\hline & & & Stage 3 & 66.66 & 33.33 & 0.13 \\
\hline & \multirow{2}{*}{ Non- AKI } & $\mathrm{RM}$ & & 28.57 & 1.28 & 0.3 \\
\hline & & NRM & & 14.28 & - & 0.1 \\
\hline
\end{tabular}

Table 4. Comparison of the mortality rate related/unrelated to the three-year recurrence between transplant patients with or without AKI based on all RIFLE, AKIN, and KDIGO criteria

\begin{tabular}{|c|c|c|c|c|c|c|}
\hline Criteria & AKI/No AKI & RM/NRM & AKI stage & Allogeneic patients' mortality (\%) & Autologous patients' mortality (\%) & $P$ value \\
\hline \multirow{8}{*}{ RIFLE } & \multirow{6}{*}{ AKI } & \multirow{3}{*}{ RM } & Risk & 14.28 & 36 & 0.27 \\
\hline & & & Injury & 33.33 & 50 & 0.7 \\
\hline & & & Failure & 33.33 & 33.33 & 0.1 \\
\hline & & \multirow{3}{*}{ NRM } & Risk & 14.28 & 22 & 0.1 \\
\hline & & & Injury & 50 & 22.22 & 0.2 \\
\hline & & & Failure & 66.66 & 33.33 & 0.12 \\
\hline & \multirow{2}{*}{ Non- AKI } & RM & & 46.66 & 13.12 & 0.14 \\
\hline & & NRM & & 26.66 & 9.37 & 0.11 \\
\hline \multirow{8}{*}{ AKIN } & \multirow{6}{*}{ AKI } & \multirow{3}{*}{ RM } & Stage 1 & 13.33 & 32.72 & 0.2 \\
\hline & & & Stage 2 & 33.33 & 47.36 & 0.12 \\
\hline & & & Stage 3 & 33.33 & 33.33 & 0.12 \\
\hline & & \multirow{3}{*}{ NRM } & Stage 1 & 13.33 & 20 & 0.2 \\
\hline & & & Stage 2 & 50 & 21.05 & 0.1 \\
\hline & & & Stage 3 & 66.66 & 33.33 & 0.1 \\
\hline & \multirow{2}{*}{ Non- AKI } & RM & & 50 & 13.56 & 0.12 \\
\hline & & NRM & & 28.57 & 9.61 & 0.2 \\
\hline \multirow{8}{*}{ KDIGO } & \multirow{6}{*}{ AKI } & \multirow{3}{*}{$\mathrm{RM}$} & Stage 1 & 12.50 & 33.92 & 0.18 \\
\hline & & & Stage 2 & 40 & 50 & 0.8 \\
\hline & & & Stage 3 & 33.33 & 33.33 & 0.1 \\
\hline & & \multirow{3}{*}{ NRM } & Stage 1 & 16.66 & 26.78 & 0.17 \\
\hline & & & Stage 2 & 60 & 25 & 0.2 \\
\hline & & & Stage 3 & 66.66 & 33.33 & 0.12 \\
\hline & \multirow{2}{*}{ Non- AKI } & $\mathrm{RM}$ & & 50 & 12.82 & 0.23 \\
\hline & & NRM & & 14.28 & .05 & 0.13 \\
\hline
\end{tabular}


was $60.27 \%$ and $22.5 \%$, respectively, which indicates no statistically significant difference by ANOVA (Table 4).

\section{Discussion}

BMT is a well-known treatment for malignant and non-malignant diseases. However, it is associated with acute and chronic side effects. The occurrence of AKI, defined as doubling $\mathrm{sCr}$ from the baseline, is the most important complication following transplantation, which has irreversible clinical consequences (10, 17, 18). Determination of the Incidence of AKI depends on the validity of the transplantation center and the early identification of these patients. According to new descriptions of AKI, it is necessary to agree on the definition of AKI following BMT. Today, there are three major definitions for AKI (19). To standardize one of the post-transplant descriptions, a definition more strongly associated with the clinical outcome should be identified (20). For this purpose, in the present study, the Incidence of AKI was calculated based on three common definitions of AKI, and patient survival was then calculated according to each of these descriptions. In this research, the incidence of AKI was $35.42 \%$ and $37.26 \%$ according to the three definitions of RIFLE as well as AKIN and KDIGO criteria, respectively. In the early years of BMT in the 1980s and 1990s, criteria like the threefold increase in sCr were used to define AKI. As a result, up to $50 \%$ of patients had acute renal failure after transplantation, but this rate decreased to $33 \%$ in the following decades. Additionally, the general occurrence of AKI in different centers is estimated at 30\%$40 \%$ today (18-20).

In the present study, the percentage of AKI incidence was generally $35.42 \%$ according to the RIFLE criteria and $37 / 26 \%$ based on the AKIN and KDIGO criteria. In this study, the occurrence of AKI after both autologous and allogeneic transplants is quite different. Some studies, including that of Parikh et al, reported up to 50\% incidence of AKI in transplant patients (10). Furthermore, in the study by Ando et al, 19\% of autologous transplant patients had AKI after BMT $(18,19)$. The frequency of AKI following autologous transplantation depends on several factors, and a higher prevalence of it in the present study is likely due to the type of transplant patients compared to the study by Ando et al, in which only $30 \%$ of patients

Table 5. Comparison of a the one-year survival rate between transplant patients with or without AKI based on all RIFLE, AKIN, and KDIGO criteria

\begin{tabular}{|c|c|c|c|c|c|}
\hline Criteria & AKI & Stages & Allogenic \% & Autologous \% & $P$ value \\
\hline \multirow{4}{*}{ RIFLE } & \multirow{3}{*}{ AKI } & $\mathrm{R}$ & 85.71 & 92 & 0.11 \\
\hline & & 1 & 66.66 & 80 & 0.13 \\
\hline & & $\mathrm{F}$ & - & 33.33 & 0.7 \\
\hline & Non-AKI & & 2.58 & 96.87 & 0.10 \\
\hline \multirow{4}{*}{ AKIN } & \multirow{3}{*}{ AKI } & 1 & 86.66 & 92.72 & 0.1 \\
\hline & & 2 & 66.66 & 78.94 & 0.15 \\
\hline & & 3 & - & 33.33 & 0.2 \\
\hline & Non-AKI & & 2.21 & 96.79 & 0.11 \\
\hline \multirow{4}{*}{ KDIGO } & \multirow{3}{*}{ AKI } & 1 & 81.25 & 87.50 & 0.2 \\
\hline & & 2 & 40 & 77.77 & 0.12 \\
\hline & & 3 & - & 33.33 & 0.7 \\
\hline & Non-AKI & & 2.95 & 98.71 & 0.12 \\
\hline
\end{tabular}

Table 6. Comparison of a three-year survival rate between transplant patients with or without AKI based on all RIFLE, AKIN, and KDIGO criteria

\begin{tabular}{|c|c|c|c|c|c|}
\hline Criteria & AKI & Stages & Allogenic \% & Autologous \% & $P$ value \\
\hline \multirow{4}{*}{ RIFLE } & \multirow{3}{*}{ AKI } & $\mathrm{R}$ & 71.42 & 42 & 0.15 \\
\hline & & I & 16.66 & 35 & 0.7 \\
\hline & & $\mathrm{F}$ & - & 33.33 & 0.1 \\
\hline & Non-AKI & & 26.66 & 77.50 & 0.51 \\
\hline \multirow{4}{*}{ AKIN } & \multirow{3}{*}{ AKI } & 1 & 73.33 & 47.27 & 0.16 \\
\hline & & 2 & 16.16 & 31.57 & 0.5 \\
\hline & & 3 & - & 33.33 & 0.1 \\
\hline & Non-AKI & & 21.42 & 76.92 & 0.48 \\
\hline \multirow{4}{*}{ KDIGO } & \multirow{3}{*}{ AKI } & 1 & 62.5 & 39.28 & 0.16 \\
\hline & & 2 & - & 27.77 & 0.14 \\
\hline & & 3 & - & 33.33 & 0.1 \\
\hline & Non-AKI & & 35.71 & 80.12 & 0.52 \\
\hline
\end{tabular}


Table 7. The prevalence of underlying diseases in patients requiring bone marrow transplantation

\begin{tabular}{lccc}
\hline $\begin{array}{l}\text { Autologous/allogeneic } \\
\text { BMT }\end{array}$ & $\begin{array}{c}\text { Underlying } \\
\text { malignancies }\end{array}$ & No. & $\%$ \\
\hline Allogeneic & AML & 30 & 11.07 \\
& ALL & 8 & 2.95 \\
Autologous & M.M & 90 & 33.21 \\
& HL & 84 & 31 \\
& NHL & 55 & 21 \\
& GCT & 4 & 1.47 \\
& Total & 271 & 100.0 \\
\hline
\end{tabular}

had lymphoma, and most of them had multiple myeloma $(11,18)$.

Numerous malignancies can lead to BMT due to treatment failure. The most common underlying disease requiring BMT is multiple myeloma (MM), which accounts for approximately one-third of the causes that require transplantation. Hodgkin's lymphoma (HL) with $31 \%$ and NHL with $21 \%$ is the next common cause for BMT, respectively. It should be noted that some relapse mortality (RM) after BMT may be due to these malignancies. In this study, underlying malignancies requiring autologous BMT included MM (33.21\%), HL (31\%), and NHL (21\%), and underlying malignancies requiring allogeneic BMT included acute myelogenous leukemia (11.07\%) and acute lymphoblastic leukemia (less than 3\%). During the study period, only four patients with testicular germ cell tumors (GCTs) underwent autologous BMT (Table 7). In this research; however, patients were transplanted because of lymphoma who received a variety of chemotherapy drugs, especially platin compounds, compared to multiple myeloma patients before transplantation. This may contribute to their susceptibility to AKI following transplantation. There were no significant differences between the three definitions for the AKI diagnosis, indicating that any of the AKI identification criteria can be used to classify AKI severity depending on the conditions of the transplant center. On the other hand, one- and three-year mortality rates were the same on all three criteria in allogeneic and autologous transplantation patients, and none of the criteria was superior to the other. In autologous transplantation, $77.5 \%$ of patients with no history of AKI survived after three years. While $39.73 \%$ of those having a history of AKI survived which indicated these results are complementary to the threeyear mortality rate (Tables 3 and 4). For instance, the three-year mortality rate in autologous transplant patients with and without a history of AKI was $60.27 \%$ and $22.5 \%$, respectively. Obviously, the three-year survival rate in autologous transplant patients with and without a history of AKI was $39.73 \%$ and $77.5 \%$, respectively. Approximately the same results were obtained for the AKIN and KDIGO criteria, which showed that AKI incidence is comparable in the three-year outcome.

The innovation of our study was a preliminary classification of the patients into dependent and nondependent relapse groups, mainly eliminating the role of underlying disease that causes the death of patients. It is important to note that there are many causes for mortality related/unrelated to the one-year relapse of BMT patients, such as ischemic acute tubular necrosis, infarction, thrombotic microangiopathy, antibodymediated rejection, ischemia, drug-induced interstitial nephritis, endothelial damage, acute calcineurin inhibitor toxicity, and fulminant disease recurrence. Accordingly, about $22 \%$ of autologous transplant patients with nonrelapse mortality (NRM) had a history of AKI. In contrast, only $9 \%$ of those patients, without a history of AKI, were associated with NRM causes, which is statistically significant.

Although the various studies show that AKI in autologous transplant patients is associated with higher mortality, it was the opposite in allogeneic BMT. The cause of the different mortality rates of the present study compared to other researches (such as Ando's and Parikh's studies) is also due to the same recurrence-related deaths. Besides, due to the low volume of allogeneic transplant patients in this study, it is not possible to discuss the relationship between mortality rate and AKI occurrence precisely.

\section{Conclusion}

Overall, the use of any of the definitions of AKI in the post-BMT period is essential for the rapid identification of patients and appropriate intervention. These patients are at worse clinical outcomes within the three years following transplantation. Due to the deterioration of the patient's conditions with AKI, it can be stated that the development of AKI after transplantation has a poor prognosis, but identifying the severity of AKI through any of the three AKI definitions (RIFLE, AKIN, and KDIGO criteria) will be useful in controlling and improving patients' conditions. Given the role of AKI in the poor prognosis of these patients, other methods are needed to identify AKI in future studies and investigate different approaches for the diagnosis of AKI in transplant patients.

\section{Limitations of the study}

Our research also had some limitations as follows. First, this research was performed within the first five years of the transplantation center, which caused some of the recorded defects in the case to influence the conclusions. Second, it is difficult to accurately record the volume of urine output, especially in transplant patients who do not have an internal catheter. Third, it is a retrospective study that has its limitations and may fail to identify patients' selection biases. Finally, the investigation of a single center 
in this study can affect its outcome.

\section{Authors' contribution}

MHG conceived the manuscript and revised it. AA and MoM wrote the manuscript and prepared tables. AH, $\mathrm{MaM}$, and $\mathrm{HK}$ were included in preparing the concept and design. MoM critically evaluated the intellectual contents. All authors participated in preparing the final draft of the manuscript, revised the manuscript, and critically evaluated the intellectual contents. All authors have read and approved the manuscript's content and confirmed the accuracy or integrity of any part of the work.

\section{Conflicts of interest}

The authors declare that they have no known competing interests or personal relationships that could have appeared to influence the work reported in this paper.

\section{Ethical considerations}

Ethical issues (including plagiarism, data fabrication, double publication) have been completely observed by the authors.

\section{Funding/Support}

This study was supported by Shahid Beheshti University of Medical Sciences, (Grant\#UNRC.931223.23).

\section{References}

1. Sawinski D. The kidney effects of hematopoietic stem cell transplantation. Adv Chronic Kidney Dis. 2014;21:96-105. doi: 10.1053/j.ackd.2013.08.007

2. Eapen M, Rubinstein P, Zhang M-J, Stevens C, Kurtzberg J, Scaradavou A, et al. Outcomes of transplantation of unrelated donor umbilical cord blood and bone marrow in children with acute leukaemia: a comparison study. Lancet. 2007;369:1947-54. doi: 10.1016/S0140-6736(07)60915-5.

3. Valette X, du Cheyron D. A critical appraisal of the accuracy of the RIFLE and AKIN classifications in defining "acute kidney insufficiency" in critically ill patients. J Crit Care. 2013;28:116-25. doi: 10.1016/j.jcrc.2012.06.012.

4. Zager R, O’quigley J, Zager B, Alpers C, Shulman H, Gamelin L, et al. Acute renal failure following bone marrow transplantation: a retrospective study of 272 patients. Am J Kidney Dis 1989;13(3):210-6. doi: 10.1016/s02726386(89)80054-x.

5. Hingorani SR, Guthrie K, Batchelder A, Schoch G, Aboulhosn N, Manchion J, et al. Acute renal failure after myeloablative hematopoietic cell transplant: incidence and risk factors. Kidney Int. 2005;67:272-7. doi: 10.1111/j.15231755.2005.00078.x.

6. Hingorani S, Guthrie K, Schoch G, Weiss N, McDonald G. Chronic kidney disease in long-term survivors of hematopoietic cell transplant. Bone marrow transplant. 2007;39:223-9. doi: 10.1038/sj.bmt.1705573.
7. Weiss A, Sandmaier B, Storer B, Storb R, McSweeney P, Parikh C. Chronic kidney disease following non-myeloablative hematopoietic cell transplantation. Am J Transplant. 2006;6:89-94. doi: 10.1111/j.1600-6143.2005.01131.x.

8. Kemmner S, Verbeek M, Heemann U. Renal dysfunction following bone marrow transplantation. J Nephrol. 2017;30:201-9. doi: 10.1007/s40620-016-0345-y.

9. Hahn T, Rondeau C, Shaukat A, Jupudy V, Miller A, Alam $\mathrm{A}$, et al. Acute renal failure requiring dialysis after allogeneic blood and marrow transplantation identifies very poor prognosis patients. Bone marrow transplant. 2003;32:405-10. doi: $10.1038 /$ sj.bmt.1704144.

10. Parikh CR, McSweeney PA, Korular D, Ecder T, Merouani A, Taylor J, et al. Renal dysfunction in allogeneic hematopoietic cell transplantation. Kidney Int. 2002;62:566-73. doi: 10.1046/j.1523-1755.2002.00455.x.

11. Merouani A, Shpall EJ, Jones RB, Archer PG, Schrier RW. Renal function in high dose chemotherapy and autologous hematopoietic cell support treatment for breast cancer. Kidney Int. 1996;50:1026-31. doi: 10.1038/ki.1996.405.

12. Kellum JA. Diagnostic criteria for acute kidney injury: present and future. Crit Care Clin. 2015;31:621-32. doi: 10.1016/j.ccc.2015.06.001.

13. Bellomo R, Ronco C, Kellum JA, Mehta RL, Palevsky P. Acute renal failure-definition, outcome measures, animal models, fluid therapy and information technology needs: the Second International Consensus Conference of the Acute Dialysis Quality Initiative (ADQI) Group. Crit Care. 2004;8:R204-12. doi: $10.1186 / \mathrm{cc} 2872$.

14. Mehta RL, Kellum JA, Shah SV, Molitoris BA, Ronco C, Warnock DG, et al. Acute Kidney Injury Network: report of an initiative to improve outcomes in acute kidney injury. Crit Care. 2007;11:R31. doi: 10.1186/cc5713.

15. Fujii T, Uchino S, Takinami M, Bellomo R. Validation of the Kidney Disease Improving Global Outcomes criteria for AKI and comparison of three criteria in hospitalized patients. Clin J Am Soc Nephrol: CJASN. 2014;9:848-854. doi: 10.2215/CJN.09530913.

16. Luo X, Jiang L, Du B, Wen Y, Wang M, Xi X. A comparison of different diagnostic criteria of acute kidney injury in critically ill patients. Crit Care. 2014;18:R144.

17. Kellum JA, Lameire N, Group KAGW. Diagnosis, evaluation, and management of acute kidney injury: a KDIGO summary (Part 1). Crit Care. 2014;18:R144. doi: 10.1186/cc13977.

18. Ando M, Mori J, Ohashi K, Akiyama H, Morito T, Tsuchiya $\mathrm{K}$, et al. A comparative assessment of the RIFLE, AKIN and conventional criteria for acute kidney injury after hematopoietic SCT. Bone Marrow Transplant. 2010;45:142734. doi: 10.1038/bmt.2009.377.

19. Parikh CR, Sandmaier BM, Storb RF, Blume KG, Sahebi F, Maloney DG, et al. Acute renal failure after nonmyeloablative hematopoietic cell transplantation. J Am Soc Nephrol. 2004;15:1868-1876. doi: 10.1097/01. asn.0000129981.50357.1c.

20. Uchino S, Bellomo R, Goldsmith D, Bates S, Ronco C. An assessment of the RIFLE criteria for acute renal failure in hospitalized patients. Crit Care Med. 2006;34:1913-17. doi: 10.1097/01.CCM.0000224227.70642.4F.

Copyright ( $) 2021$ The Author(s); Published by Nickan Research Institute. This is an open-access article distributed under the terms of the Creative Commons Attribution License (http://creativecommons.org/licenses/by/4.0), which permits unrestricted use, distribution, and reproduction in any medium, provided the original work is properly cited. 